\title{
Analysis of Signal Integrity for High Precision Digital-to-Analog Conversion Circuit
}

\author{
Jing Dai and Yanmei Wang \\ College of automation and electrical engineering, Shenyang Ligong University, \\ Shenyang, 110159, China \\ daijing6615@163.com,wangymsit@hotmail.com
}

\begin{abstract}
In order to improve the accuracy of the digital to analog conversion (DAC), the reflection and crosstalk problems of the signal transmission process were studied in this paper. The Hyperlynx software was used for the simulation study of reflection and crosstalk problems of the master clock signal in the DAC circuit. Source end cascading termination resistor and remote end termination resistor and capacitor $(R C)$ methods were adopted to weaken the reflected signal. The problem of signal crosstalk was solved by the remote end cascading termination resistor method. The experiments results showed that the reflection peak could be eliminated when the value of the source end

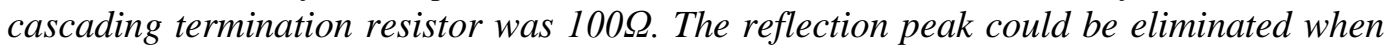
the value of remote end termination resistors was $100 \Omega$ and the capacitance was $300 \mathrm{pF}$.

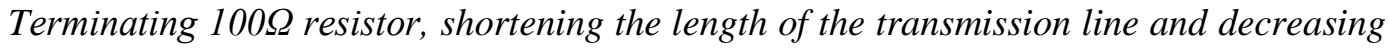
the distance of the adjacent network could weaken crosstalk phenomenon. In high precision digital to analog conversion circuit, the source end cascading termination resistor and the remote $R C$ termination were adopted to solve the problems of signal reflection. The methods of optimizing wiring and the cascading termination resistor weakened the signal crosstalk phenomenon. They also improved signal integrity and $A D C$ accuracy of the signal during transmission.
\end{abstract}

Keywords: Signal integrity, Digital-to-Analog conversion, Reflection, Crosstalk, Cascading termination

\section{Introduction}

With the development of science and technology, the micro-positioning and micromanipulation have become important parts of modern science and technology. In the high-precision control system of micro- positioning platform, analog to digital conversion (ADC) circuit and digital to analog conversion (DAC) circuit are proposed to a higher quality on account of the system which should be high-precision and real-time. With the rapid development of modern electronic design and chip manufacturing technology, circuit design tends to a new aim which the circuit pattern complexity, component layout optimization, high wiring density and high clock frequency. At the same time, the conversion accuracy and conversion speed of ADC and DAC is also increasing [1]. To ensure the accuracy of micro-positioning platform, the 24 bit ADC was adopted. Because of the impedance mismatch and other reasons for the printed circuit board, a series of questions such as reflection and crosstalk appeared in the process of ADC and DAC, which affected the signal integrity [2-3]. The signal deviated from the actual value and appeared distortion, which couldn't achieve the anticipative conversion accuracy. To ensure the integrity of the signal, we must control signal reflection and crosstalk, make the signal distortion maintain within the range of permissible. Otherwise, the noise of transmission line and signal reflection will cause data errors or timing disorder. We can analyze and process the signal reflection and crosstalk with the Hyperlynx software [4]. 
In this paper, we analyze and process the signal reflection and crosstalk problems of DAC circuit for micro-positioning control system based on the Hyperlynx software.

\section{Design of DAC Circuit}

Figure 1 shows the DAC circuit for the micro-positioning control system. The core processor of control system is TMS320C6713 DSP chip manufactured by Texas Instruments (TI), DAC chip is PCM3168A audio codec chip with 24-bit accuracy also manufactured by TI. PCM3168A contains the operating mode register. DSP processor can control the operating mode of PCM3168A by SPI or $\mathrm{I}^{2} \mathrm{C}$ communication. PCM3168A supports $8 \mathrm{kHz}$ to $192 \mathrm{kHz}$ sampling rates, the signal to noise ratio can reach $112 \mathrm{~dB}$, and it integrates analog low-pass filter inside. PCM3168A also supports a variety of formats of data input, including $\mathrm{I}^{2} \mathrm{~S}$ format, Left-Justified format, Right-Justified format, DSP format and TDM format. TDM format was used to realize the serial data input in this circuit. 5V operating voltage was provided for the analog part of PCM3168A, and 3.3V operating voltage was provided for the digital part.

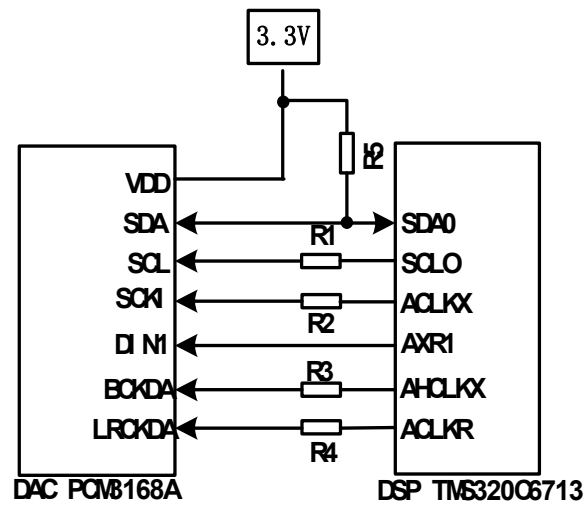

Figure 1. The Diagram of DAC Circuit

The TMS320C6713 DSP chip outputs the digital signals through McASP channel. Its [AXR1] pin connects with PCM3168A's [DIN1] pin. The PCM3168A was configured serial digital signal input mode. The clock signal of DA converter was provided by TMS320C6713 DSP chip and transmitted through McASP channel of DSP chips. The [SDA] and [SCL] pins of PCM3168A chip connect with [SDA0] and [SCL0] pins of TMS320C6713 DSP chip based on the $\mathrm{I}^{2} \mathrm{C}$ communication mode, which could realize the configuration of the operating mode of register inside PCM3168A chip. The bidirectional serial data was transmitted by [SDA0] pin. The clock signal is transmitted by [SCL0] pin. The $[\mathrm{SCKI}]$ pin of PCM3168A is an input pin for master clock signal, which connects with DSP's [ACLKX] pin. The [BCKDA] pin is an input pin of bit clock signal, which connects with TMS320C6713 DSP chip's [AHCLKX] pin. The [LRCKDA] pin is an input pin of word clock signal, which connects with TMS320C6713 DSP chip's [ACLKR] pin. It's required to strictly obey the setting clock when data is transmitted. A word clock period contains 48 bit clock periods, and a bit clock corresponds to a bit data transmitting.

As shown in Figure 1, the crosstalk and reflection about the master clock signal of [SCKI] pin were analyzed in this paper. The pin which outputs clock signal from DSP chip is source-end, and the pin which receives clock signal of the DAC chip is remoteend. The analysis about the integrity of clock signal of source-end and remote-end would obtain the best answer to solve the problem about signal reflection and crosstalk. 


\section{Research on the Problem of Signal Reflection}

\subsection{Theory of Signal Reflection}

When the signal propagates along the transmission line, it will be impacted by the transient impedance all the times. The impedance maybe comes from the transmission line, and also it could come from other components on load end. If the impedance is constant, the signals will propagate forward normally. If the impedance changes, the signal reflection will occur. Moreover, other factors such as corner of transmission line, via hole of print circuit board (PCB), T-type wire, connector and packaging of transmission line could cause reflection. Figure 2 shows a model of signal reflection [5]. The input voltage signal $\mathrm{V}_{\mathrm{i}}$ transmits along the transmission line. When the impedance of transmission line changes from $Z_{1}$ to $Z_{2}$, a part of the $V_{i}$ is reflected back, and the reflected voltage is represented by $V_{r}$. The rest of the voltage $V_{i}$ transmits along the transmission line and is represented by transmission voltage $\mathrm{V}_{\mathrm{t}}$. The reflection coefficient ( $\rho$ ) can be expressed by formula (1). The transmission coefficient (t) can be expressed by formula (2).

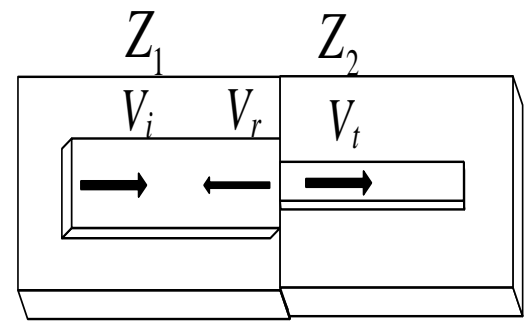

Figure 2. Model of Signal Reflection

$$
\begin{array}{r}
\rho=\frac{V_{r}}{V_{i}}=\frac{Z_{2}-Z_{1}}{Z_{2}+Z_{1}} \\
t=\frac{V_{t}}{V_{r}}=\frac{2 Z_{2}}{Z_{1}+Z_{2}}
\end{array}
$$

When impedance is matching namely $\mathrm{Z}_{1}=\mathrm{Z}_{2}$, reflected voltage and reflection coefficient are all zero, and transmission voltage $V_{t}$ is equal to the input voltage $V_{i}$ by formula (1) and formula (2). It is showed that all the input voltage signals are transmitted to the termination of load. When the termination is an open circuit namely $Z_{2}=\infty$, reflected voltage $V_{r}$ is equal to the input voltage $V_{i}$, transmission voltage $V_{t}$ is equal to $2 V_{i}$. When the termination shorts namely $Z_{2}=0$, the reflected voltage $V_{r}$ will be generated on the termination, and it is equal and opposite with the input voltage $V_{i}$ and returns to the source end. At the moment, the reflected voltage $V_{r}$ is equal to the input voltage $V_{i}$ of opposite direction, the transmission voltage $V_{t}$ is zero.

According to the principle of reflection, impedance matching is the fundamental way to solve reflection problem, which can be done by the way of terminating resistor or capacitor to achieve matching [6]. Terminating resistor or capacitor is a general technique to achieve impedance matching, which can reduce the reflection due to the impedance mismatch between source end and load end. Termination can be realized through the following four ways: source end cascading termination, remote parallel termination, remote Thevenin termination and remote RC termination [7]. In this paper, source end cascading termination and remote $\mathrm{RC}$ termination experiment are discussed. 


\subsection{Simulation and Processing of Master Clock Signal Reflection}

The reflection simulation of electronic components was studied based on IBIS (I/O Buffer Information Specification) model for PCB. The master clock signals input to the digital to analog converter PCM3168A chip through the [SCKI] pin.

When the master clock signals run into reflection and crosstalk in the process of transmission, the conversion accuracy of digital to analog converter will be reduced. The waveform of the master clock signals through the [SCKI] pin was shown in Figure 3, which was impacted by reflection signal without the terminating resistor.

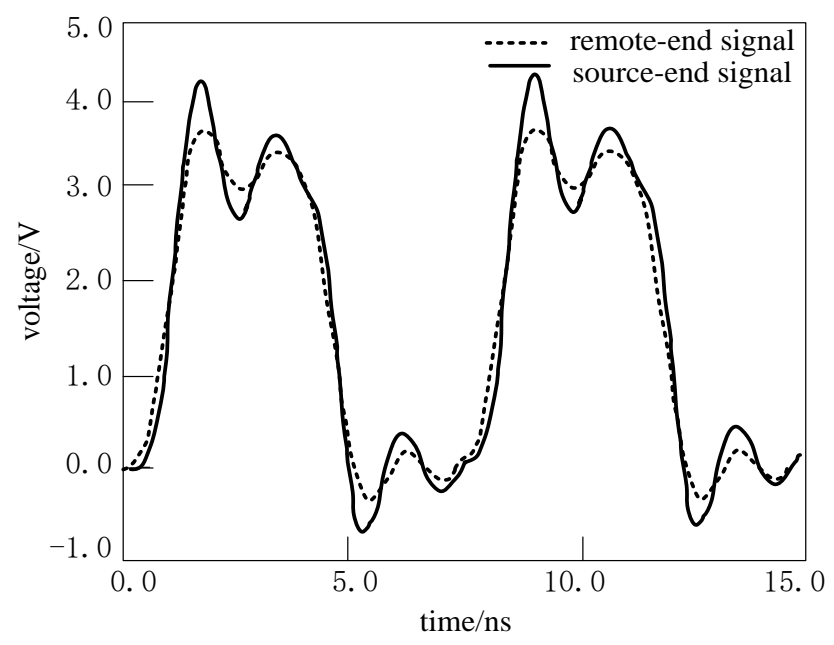

Figure 3. The Master Clock Signal Waveform without Terminating Resistor

In Figure 3, the solid line shows the source end master clock signal waveform from [ACLKX] pin of DSP, and the dashed line shows the remote master clock signal waveform received by [SCKI] pin. As shown in the Figure 3, the source and remote end signals does not coincide, there are signal fluctuations and the emergence of the peak. Obviously, both of source and remote end signals have been impacted. The overshoot voltage of source end signals runs up to $4.2 \mathrm{~V}$, the undershoot voltage reaches $-0.6 \mathrm{~V}$. The overshoot voltage of remote end received signal runs up to $3.7 \mathrm{~V}$, the undershoot voltage reaches $-0.4 \mathrm{~V}$. There are high overshoot and undershoot in the process of signal transmission, and the signal distortion is very apparent. It is indicated that signal integrity is destructive. In this paper, two kinds of methods of terminating to eliminate reflection were studied, the source end cascading termination resistor, and the remote end termination resistor and capacitor.

\subsubsection{Source End Cascading Termination Resistor}

As shown in the Figure 4, source end cascading termination resistor means that the resistance is cascaded with the source end [8]. When the value of source end cascading termination resistor of master clock transmission line is $50 \Omega$, the reflection simulation waveform is shown in Figure 5. Compared with Figure 3, after terminating $50 \Omega$ resistor, spike voltage amplitude of the source end signals decreased from $4.2 \mathrm{~V}$ to $3.7 \mathrm{~V}$, spike voltage amplitude of remote signal decreased from $3.7 \mathrm{~V}$ to $3.5 \mathrm{~V}$, fluctuation of the clock signal also had a certain improvement. 


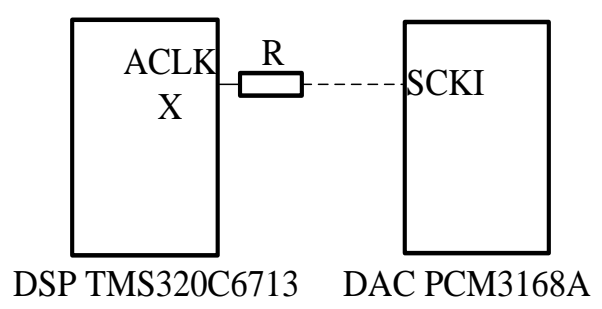

\section{Figure 4. The Diagram of Source End Cascading Termination Resistor}

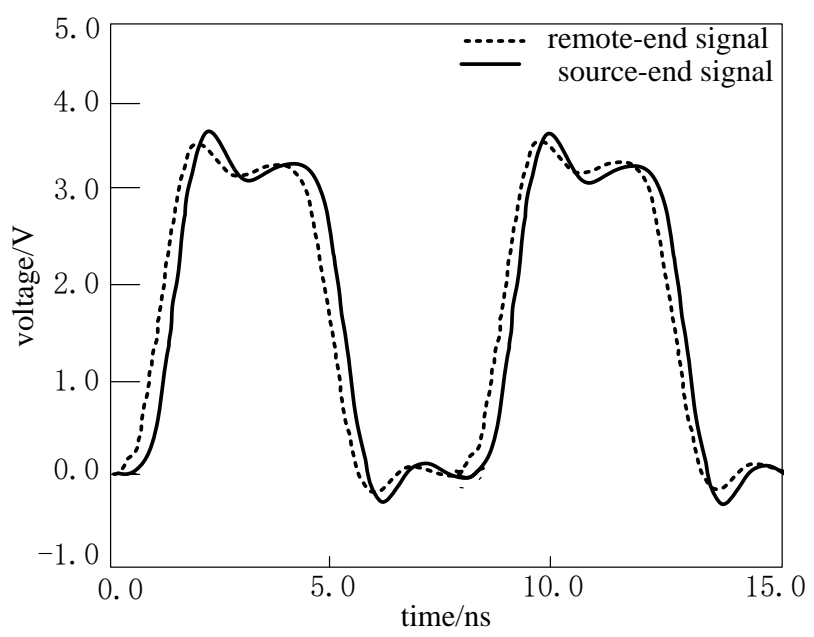

Figure 5. The Reflection Simulation Waveform of Source End Cascading Termination $50 \Omega$ Resistor

Figure 6 shows the reflection simulation diagram of source end cascading termination $100 \Omega$ resistor when the value of terminating resistor was changed into $100 \Omega$ in Figure 4.

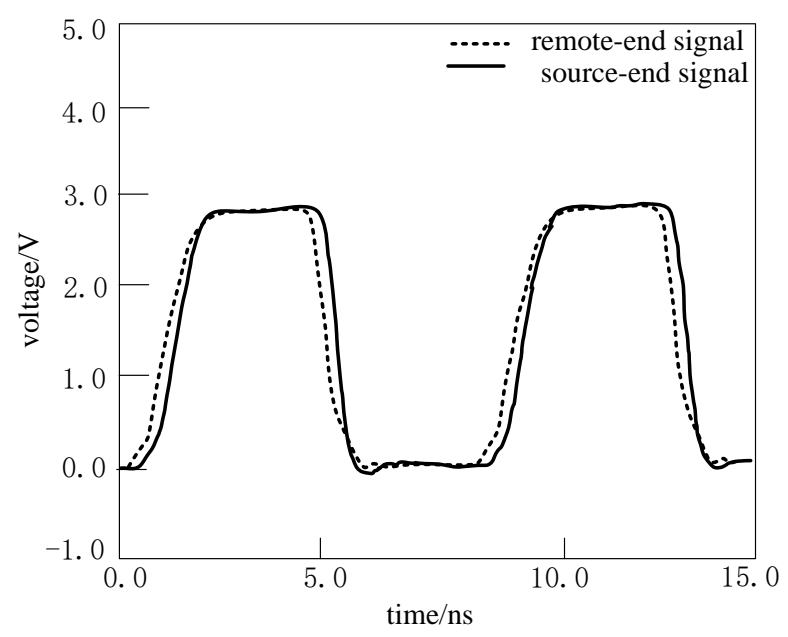

Figure 6. The Reflection Simulation Diagram of Source End Cascading Termination $100 \Omega$ Resistor

As shown in Figure 6, the spikes voltage of source end and remote clock signals are all eliminated basically, the waveforms of source end and remote end are coincided approximately, fluctuation of signal is decreased, and reflection phenomenon is eliminated obviously when the value of terminating resistor was changed into $100 \Omega$. 


\subsubsection{Remote End Termination Resistor and Capacitor (RC)}

RC remote matching is widely applied in the system of TTL and CMOS [9]. Terminating impedance should match the impedance of the transmission lines, the capacitance is generally between 100 to $600 \mathrm{pF}$. Although the DC power consumption of the termination resistor and capacitor is very low, the method of RC termination could provide high quality signals. A disadvantage for RC termination is that the rising and falling time of remote signal will be prolonged because of capacitive load to increase the signal delay. This method is not suitable for high frequency signal. In this design, the impedance of transmission line is about $75 \Omega$ in an ideal state. But in reality, the impedance of transmission line is about $100 \Omega$ because it is affected by the dielectric of PCB and internal resistance of driving end and load end [10]. Figure 7 shows the diagram of remote RC termination. Figure 8 shows the reflection simulation diagram of terminating $100 \Omega$ resistor and $100 \mathrm{pF}$ capacitor. Compared with figure 3 , it can be found that spike voltage of source end and remote signal was basically eliminated, and the clock signal fluctuation was reduced. The overshoot voltage of source end signal decreased from $4.2 \mathrm{~V}$ to $3 \mathrm{~V}$ and the undershoot voltage decreased from $-0.6 \mathrm{~V}$ to $0 \mathrm{~V}$, the overshoot voltage of remote signal decreased from $3.7 \mathrm{~V}$ to $2.9 \mathrm{~V}$ and the undershoot voltage decreased from $-0.4 \mathrm{~V}$ to $-0.1 \mathrm{~V}$, the rising edge turned slow. There was strong ringing in the trough of wave.

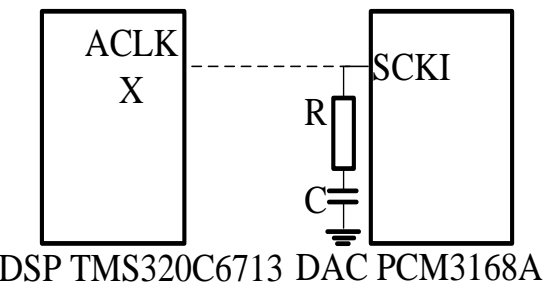

\section{Figure 7. The Diagram of Remote RC Termination}

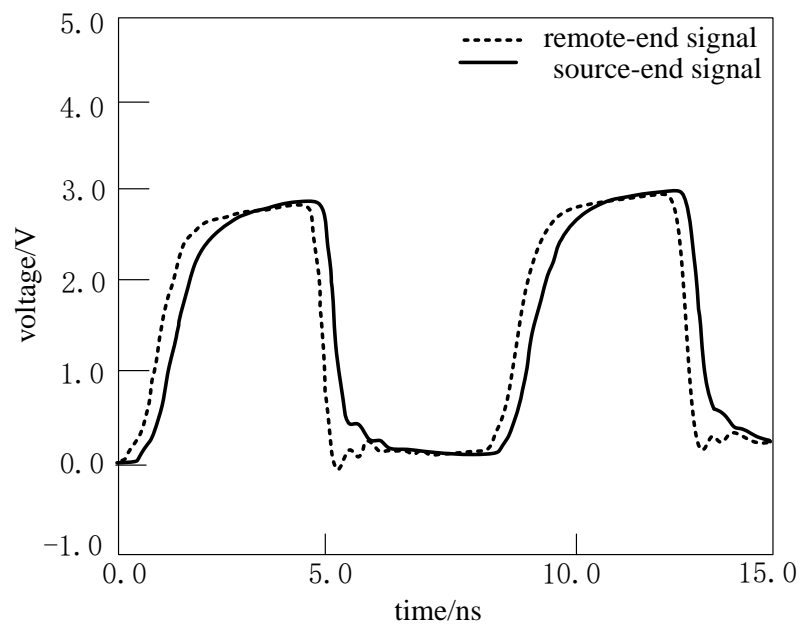

Figure 8. The Reflection Simulation Diagram of Terminating $100 \Omega$ Resistor and 100 pF Capacitor

Figure 9 shows the reflection simulation diagram of terminating $100 \Omega$ resistor and $200 \mathrm{pF}$ capacitor. It can be found that spike voltage was eliminated basically, waveforms of source end and remote were coincided approximately and the ringing in the trough of wave was disappeared basically. 


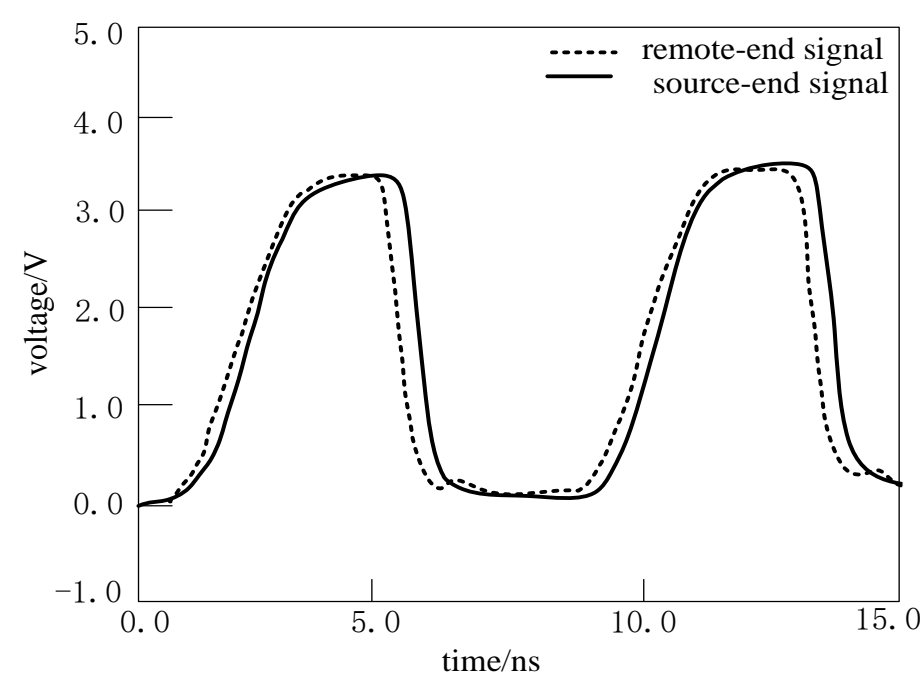

\section{Figure 9. The Reflection Simulation Diagram of Terminating $100 \Omega$ Resistor and 200pF Capacitor}

Figure 10 shows the reflection simulation diagram of terminating $100 \Omega$ resistor and $300 \mathrm{pF}$ capacitor. It can be found that spike voltage was eliminated basically, waveforms of source end and remote were coincided approximately and phenomenon of reflection was disappeared basically.

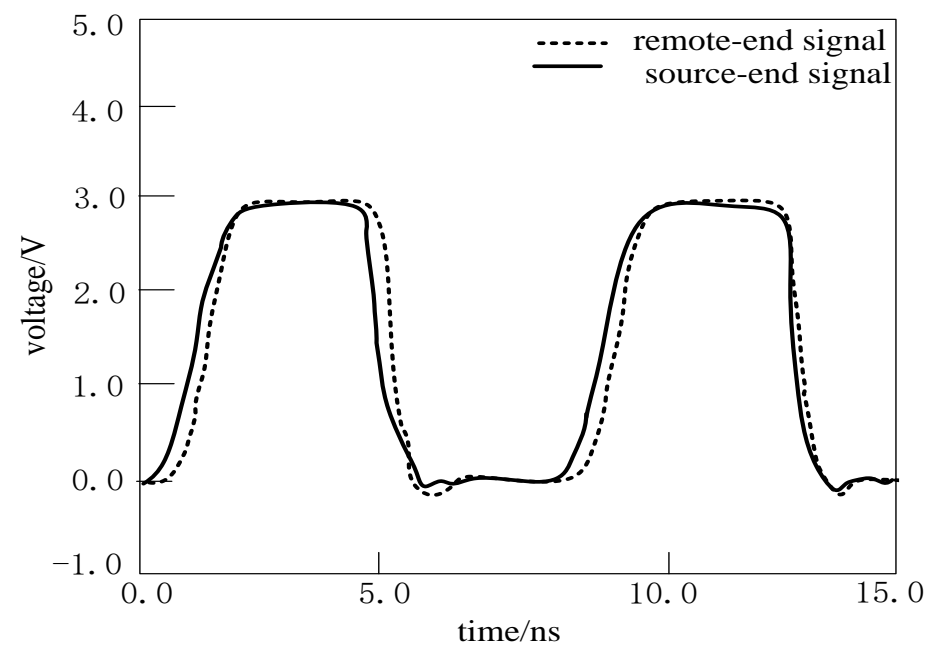

Figure 10. The Reflection Simulation Diagram of Terminating $100 \Omega$
Resistor and 300pF Capacitor

Based on the above experimental results, the appropriate way to solve the problem of reflection is terminating $100 \Omega$ resistor and $300 \mathrm{pF}$ capacitor on remote $\mathrm{RC}$ termination or terminating $100 \Omega$ resistor on source end cascading termination. Compared with two methods, it can be concluded that the method of source end cascading termination resistor is simple and practicable aiming at reflection problem. 


\section{Research on the Problem of Signal Crosstalk}

\subsection{The Theory of Signal Crosstalk}

Crosstalk is an undesirable voltage noise jamming which generated from adjacent transmission lines because of electromagnetic coupling when the signals are transmitted on transmission lines [11]. Serious crosstalk may cause false triggering and lead to malfunction of systems. Crosstalk can't be eliminated but can be weakened. The network which is affected by noise is usually called interfered-network or victim network, which expressed in $\mathrm{N}_{1}$ as shown in Figure 11 [12]. The network which is noise source is called the interference network or attack network, which expressed in $\mathrm{N}_{2}$. The major cause is mutual inductance among many causes of crosstalk. Figure 11 shows the problem of crosstalk caused by mutual inductance.

A single network is equivalent to a lot of inductors in series. That is, $1_{1}, 1_{2} \ldots \ldots 1_{\mathrm{m}}$ and $\mathrm{M}$ inductors made up the network $\mathrm{N}_{1}, \mathrm{~L}_{1}, \mathrm{~L}_{2} \ldots \ldots \mathrm{L}_{\mathrm{n}}$ and $\mathrm{M}$ inductors made up the network $\mathrm{N}_{2}$. The interaction of the two inductance networks generates mutual inductance. $\mathrm{M}$ is the mutual inductance, which marks the degree of induced current when the line $\mathrm{N}_{2}$ stimulates the line $\mathrm{N}_{1}$ by the magnetic field. Because of mutual inductance, the signal currents which passing through interference network $\mathrm{N}_{2}$ induce the noise voltage $\mathrm{V}$ in network $\mathrm{N}_{1}$ which is in the range of magnetic field of $\mathrm{N}_{2}$. The noise voltage $\mathrm{V}$ is proportional to the change of the current $I$ in the network $\mathrm{N}_{2}$. It can be calculated by formula (3) [13].

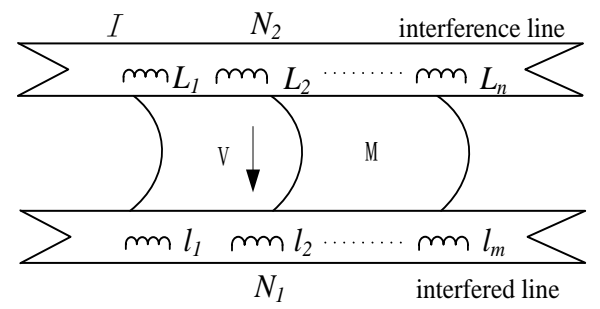

Figure 11. The Diagram of Crosstalk Caused by Mutual Inductance

$$
V=M \frac{d I}{d t}
$$

\subsection{The Simulation and Processing of Master Clock Signal Crosstalk Problem}

This paper describes the simulation analysis about master clock network crosstalk. The master clock network $\mathrm{N}_{1}$ connects [ACLKX] pin of TMS320C6713 and [SCKI] pin of PCM3168A. The network $\mathrm{N}_{2}$ with [SCL] pin of TMS320C6713 and [SCL0] pin of PCM3168A is clock input network for D/A configuration register, it will interfere with master clock network $\mathrm{N}_{1}$. Figure 12 shows the diagram of master clock crosstalk network.

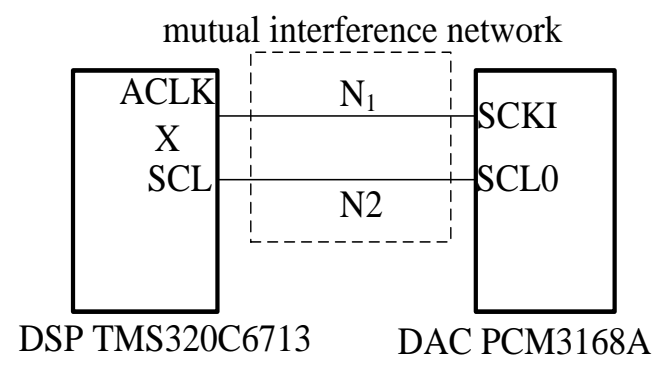

Figure 12. The Diagram of Master Clock Crosstalk Network 
If the proper measure is not taken, the interference from crosstalk is very strong. Figure 13 shows the crosstalk simulation diagram without terminating resistor. It is shown that current peak to peak value of interference network was around $2 \mathrm{~mA}$, interfered-network suffered strong interference and the current peak to peak value was around $4.4 \mathrm{~mA}$.

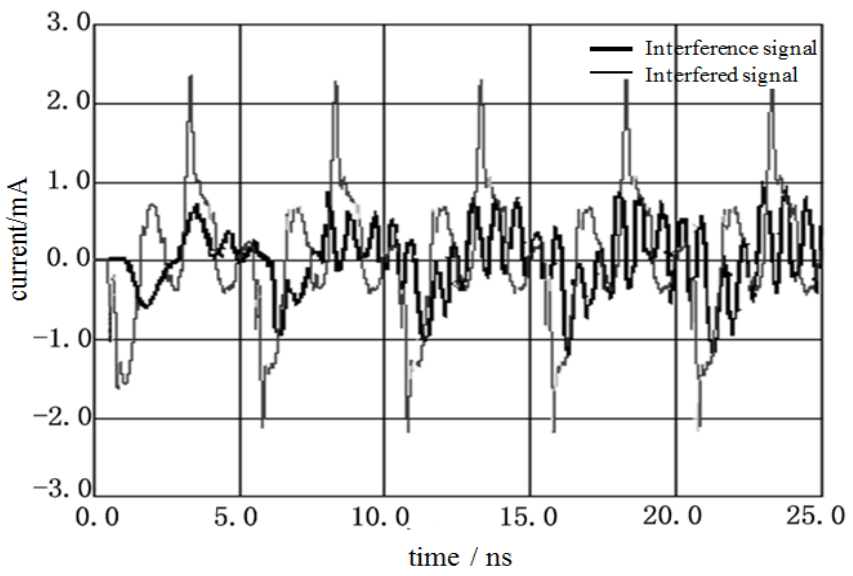

Figure 13. The Crosstalk Simulation Diagram without Terminating Resistor

There are two methods to weaken the crosstalk: optimizing PCB wiring and source end termination resistor.

a. Optimizing wiring is increasing network spacing and decreasing the transmission distance. The distance between adjacent networks was increased from 8 mils to 16 mils within the specified range. The distance of transmission line was decreased from 1.4 inch to 1 inch. Figure 14 shows the optimized crosstalk simulation diagram. The signal of interference network weakened obviously, the peak to peak value decreased from about $2 \mathrm{~mA}$ to $1.2 \mathrm{~mA}$ and peak to peak value of interfered-network decreased from about $4.4 \mathrm{~mA}$ to $3.5 \mathrm{~mA}$ compared with Figure 13 . It can be concluded that not only the interference network crosstalk signal was reduced but also the interfered-network was optimized by optimizing wiring.

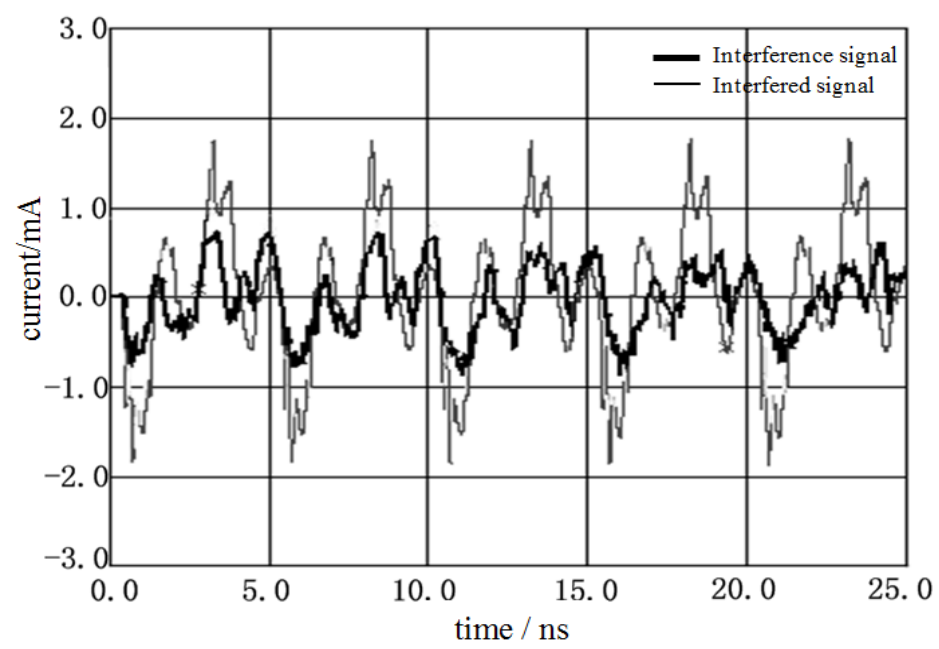

Figure 14. The Improved Crosstalk Simulation Diagram by Optimizing Wiring 
b. To further reduce the crosstalk signals and improve the quality of the signal, the method of remote cascading termination resistors was adopted. Cascading terminating resistors can reduce the sudden change of current and voltage on interference line, and ensure that the noise signal is within the range of the allowable range. Figure 15 shows the diagram of cascading terminating $100 \Omega$ resistor.

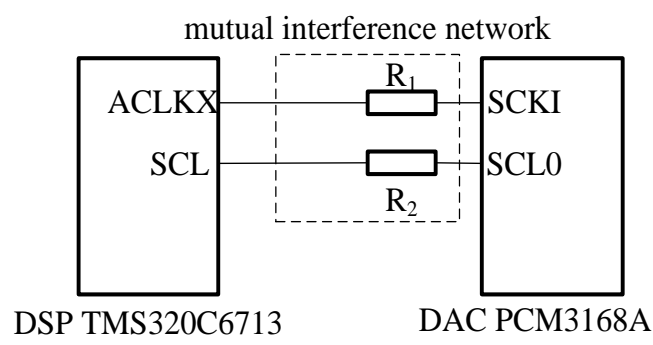

\section{Figure 15. The Diagram of Cascading Terminating $100 \Omega$ Resistor}

In Figure 15, if $R_{1}=R_{2}=100 \Omega$, the simulation diagram of crosstalk network is shown in Figure 16. The peak to peak value of interfered-network was about $2.5 \mathrm{~mA}$ and decreased from $4.4 \mathrm{~mA}$ to $2.5 \mathrm{~mA}$. The peak to peak value of interference network was around $0.5 \mathrm{~mA}$, which indicated the signal interference of adjacent network weakened obviously.

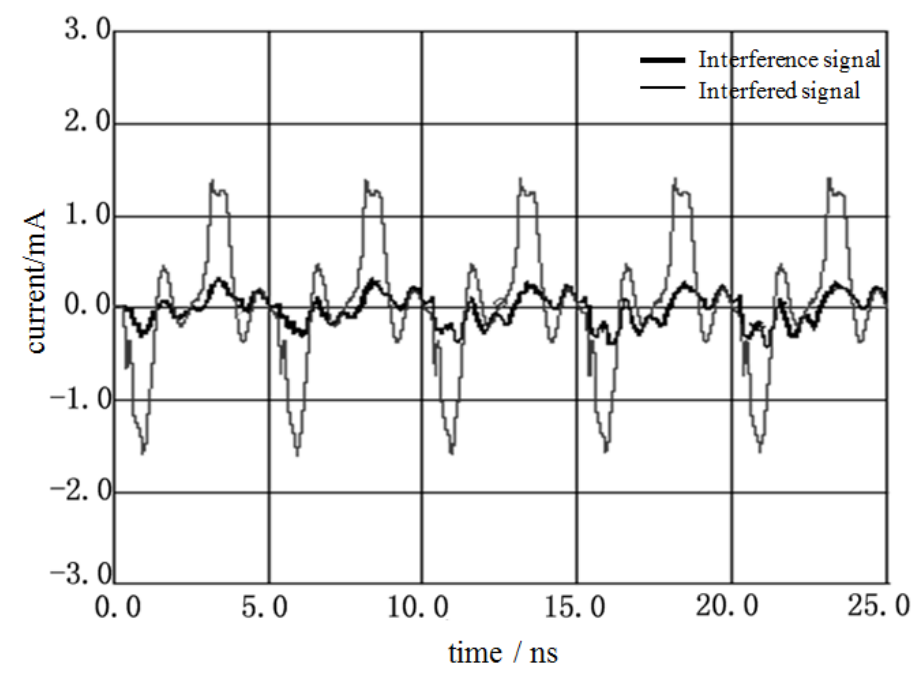

Figure 16. The Crosstalk Simulation Diagram of Terminating $100 \Omega$ Resistor

\section{Conclusion}

In this paper, the signal integrity was analyzed based on Hyperlynx software. The reflection question of master clock network and adjacent network crosstalk question were discussed for DAC circuit. Compared with two methods of terminating, it can be concluded that source end cascading termination is more appropriate than remote RC termination for reflection question. The methods of optimizing PCB wiring and the cascading termination resistor weakened the signal crosstalk phenomenon. The integrity of transmission signal on transmission line is improved. 


\section{Acknowledgements}

This work was supported by the scientific research fund of Liaoning provincial education department under project no. L2013084.

\section{References}

[1] Md. S. Ali, "The Challenges of Data Transmission toward Tbps Line rate in DWDM System for Long haul Transmission", International Journal of Future Generation Communication and Networking, vol. 7, no. 1, (2014), pp. 209-216.

[2] E. Berg, "Signal integrity analysis", Electronic Industry Press, Beijing, (2005).

[3] M. Hassad, A. S. Boughrara and T. Fortaki, "Full-wave Analysis of Rectangular Microstrip Antenna Printed on Electric-Magnetic Uniaxial Anisotropic Substrates", International Journal of Future Generation Communication and Networking, vol. 7, no. 4, (2014), pp. 183-194.

[4] X. G. Li, L.Y. Zhu and J. C. Shen, "Signal integrity simulation analysis", Instrument Technology, no. 1, (2008), pp. 41-42.

[5] Y. J. Peng, J. R. Guo and B. F. Cao, "Study for termination of reflections based on HyperLynx", Journal of Hunan University of Arts and Science, vol. 23, no. 3, (2011), pp. 50-51.

[6] D. C. Qin, L. Chen and Y. Z. Pu, "Method Research of Impedance Matching Based on Analysis of Signal Integrity", Science Technology and Engineering, vol. 8, no. 4, (2008), pp. 1051-1052.

[7] T. Ni, "High Speed Digital Circuit Signal Integrity Analysis and Simulation", Doctoral Dissertation, Xi'an Electronic and Science University, Xi'an, (2010).

[8] A. C. Scogna, A. Orlandi and V. Ricchiuti, "Signal and Power Integrity Analysis of Differential Lines in Multilayer Printed Circuit Boards With Embedded Electromagnetic Bandgap Structures", IEEE Transactions On Electromagnetic Compatibility, vol. 52, no. 2, (2010), pp. 356-357.

[9] H. F. Zhang, "HyperLynx simulation and PCB design", Electronic Industry Press, Beijing, (2005).

[10] E. Matoglu, N. Pham, D. N. Araujo, M. Cases and M. Swaminathan, "Statistical Signal Integrity Analysis and Diagnosis Methodology for High Speed Systems", IEEE Transactions On Advanced Packaging, vol. 27, no. 4, (2004), pp. 610-611.

[11] C. Li, T. H. Zhang, B. G. Chen and C. Shang, "Simulation and Improvement of Signal Integrity for DSP System Design Based on HyperLynx", Tactical Missile Technology, no. 4, (2011), pp. 111-112.

[12] A. N. Isaev, A. P. Leonov, A. V. Savel'ev, V. A. Sen'ko, A. N. Sytin and N. A. Shalanda, "Analysis of the Integrity of Signals in Data Buses by Using the System Simulation", Instrument and Experimental Techniques, vol. 48, no. 5, (2005), pp. 606-607.

[13] S. H. Jia, G. S. Dou and C. Yuan, "Analysis and Optimization of Crosstalk Based on High Speed Interconnection Signal”, Science and Technology Information, no. 4, (2010), pp 399-400.
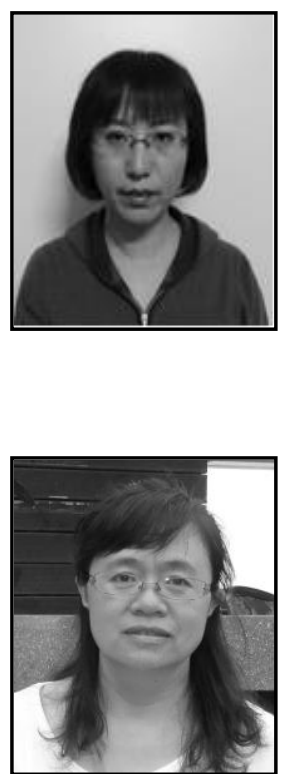

\section{Authors}

Jing Dai, she received the B.E. in Electronic Engineering from Shenyang University of Technology in 1991, and the M.S. and the Ph.D. degrees in Detection Technology and Automatic Equipment from the Northeastern University, Shenyang, China, in 1999 and 2005, respectively. Currently she serves an Associate Professor in Shenyang Ligong University, Shenyang, China. Her research interests include digital signal processing and wireless communication.

Yanmei Wang, she received her B.E. in Industry Automation from Northeastern University in 1991, and the M.S. degree in Biomedical Engineering from the Northeastern University, Shenyang, China, in 2008. Currently she serves an Associate Professor in School of Information Science and Engineering, Shenyang Ligong University. Her researches cover signal analysis and pattern recognition. 
International Journal of Future Generation Communication and Networking Vol. 9, No. 5 (2016) 